\title{
Road and Tunnel Extraction from SPOT Satellite Images Using Neural Networks
}

\author{
Nima Ghasemloo ${ }^{*}$, Mohammad Reza Mobasheri ${ }^{1}$, Ahmad Madanchi Zare ${ }^{2}$, \\ Mehran Memar Eftekhari ${ }^{2}$ \\ ${ }^{1}$ Khaje Nasireddin Toosi University of Technology, Tehran, Iran \\ ${ }^{2}$ Malek Ashtar University of Technology, Tehran, Iran \\ Email: *Nima.ghasemloo@gmail.com
}

Received November 17, 2012; revised December 20, 2012; accepted January 22, 2013

\begin{abstract}
Road extraction from the satellite images can be used in producing road maps as well as managing and developing geospatial databases. In this work, the extraction of roads from SPOT satellite images using artificial neural network has been studied. Then the location of tunnel is extracted from image using digital elevation information. Also it is tried to enhance the precision of the road extraction method using spectral information as well as texture and morphology. The method was implemented on SPOT satellite images of Tabriz and Miyaneh (Iran). The results of this research indicate that it would be possible to promote the precision of road extraction by using texture and morphology in image classification using neural networks. Finally the location of tunnel was extracted by digital elevation information. Junctions of roads and mountains have high potential in locating the tunnel. For this reason, in this study, the junctions of roads and mountains were also detected and used.
\end{abstract}

Keywords: Neural Networks; Road; Classification; Texture

\section{Introduction}

\subsection{Road and Tunnel Extraction}

The use of satellite images and their potential to be used in a wide variety of applications such as preparing and updating maps have made the automatic extraction of object, especially roads and buildings, a new challenge in remote sensing [1]. Automatic road extraction provides the means for creation, maintaining, and updating transportation networks [1]. In this study, for extracting tunnel from SPOT satellite images, the roads are extracted first then junctions of roads and mountains are detected by using digital elevation information [2]. The geology along a tunnel alignment plays a dominant role in many of the major decisions that must be made in planning, designing, and constructing a tunnel. Geology dominates the feasibility, behavior, and cost of any tunnel.

Although difficult to appreciate, the engineering properties of the geologic medium and the variations of these properties are as important as the properties of the concrete or steel used to construct the tunnel structure. In a tunnel, the ground acts not only as the loading mechanism, but also as the primary supporting medium. Thus, it is vital that the most appropriate geotechnical investi-

*Corresponding author. gation is conducted early in the planning process for any tunnel [3]. Groundwater has the potential to cause great difficulties for underground work, and a special effort should be made to define the groundwater aquifers, sources of water, water quality and temperature, depth to groundwater [4]. Vigorous methods have been proposed for automatic and semi-automatic extraction of road networks from satellite images where few of them are explained below.

\subsection{Relates Researches and Review}

A comprehensive review on the proposed methods for road extraction is found in Mena (2003) where these methods are categorized from different aspects. A comprehensive reference list is also accessible [5].

Mohammadzadeh et al. (2006) proposed a new fuzzy segmentation method for road detection in high resolution satellite images with only a few numbers of road samples [6].

Then by using an advanced mathematical morphological operator, road centerlines were extracted. A road detection strategy based on the neural network classifiers was introduced by Mokhtarzade and Valadan (2007) where a variety of input spectral parameters were tested on the functionality of the neural network for both road 
and background detection [7].

Idbraim et al. (2008) presented an automatic system of urban road extraction from satellite and aerial imageries. Their approach is based on an adaptive directional filtering and watershed segmentation [8].

Mangala (2010) et al. presented an automatic rural road extraction by using neural network from satellite image [9].

The research presented by Mena and Malpica (2005) is a good example for exploiting texture information in road extraction. In their paper, Mena and Malpica, performed a GIS updating using the pre-existing vectorial information and the RGB bands of high resolution satellite or aerial images. The binary segmentation performed in this study was based on texture progressive analysis; the three level of texture statistical evaluation being developed based on evidence theory framework [10].

Xue Dongsheng (2005) presented a technique a technique for automatic recognition of tunnel entries from super resolution remote sensing images. First, it screens the majority of irrelevant objects such as mountains and woods in remote sensing images, and extracts the areas which include potential road information from remote sensing images. Because these tasks are for reducing the area which include road seed and shortening the time for searching road, it is based on simple and quick threshold segmentation. In the potential road area, it automatically finds road seed in terms of improved randomized Hough transformation (GRHT), which is superior to randomized Hough transformation on detecting speed and detecting precision, then automatically it searches road from road seed point according to tactic of dynamic programming. Finally, it finds entries of tunnels through estimating the function of expense that has been used to search road from road seed and texture feature of mountain [11]. Junctions of roads and mountains have high potential for

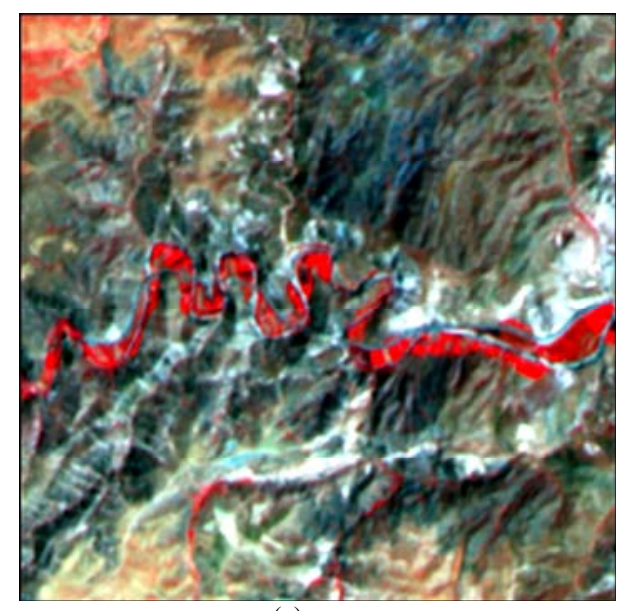

(a) the locating the tunnels. For this reason, in this study, Junctions of roads and mountains are detected.

This paper is organized as follows: In Section 2 the method for road extraction is described. In this section, the image is classified by neural network method. This method uses spectral and texture characteristics for image classification to acquire high accuracy. These characteristics are introduced to neural network as input data. When classification is done, to connect road pixels and to reduce noises due to classification, a mathematical morphological method was used and an algorithm for connect road pixels based on nearby pixels was introduced and then the short lines were deleted. After road extraction from SPOT image using elevation data, and with the help of waterways and slope data, the places of tunnels were discovered. In Section 3, results are evaluated and finally the conclusion is presented in Section 4.

To implement this work, the SPOT image from road between Tabriz and Miyaneh in Iran is used. This image is acquired in 2005 in 4 bands with spatial reference. Part of this image is shown in Figure 1.

\section{Methodology}

\subsection{Road Extraction from SPOT Image}

In order to extract road from satellite image, methods are usually depending upon the spatial resolution and sensor characteristics. In these images, roads are shown as thin lines in low resolution while in high resolution image; roads appear as homogeneous area [7]. There are different methods for image classification where neural network is one of the frequently used methods in this regard in this research, the image is classified through neural network and is divided into two categories (i.e., road and non road). In what follows, classification by neural networks method is discussed briefly.

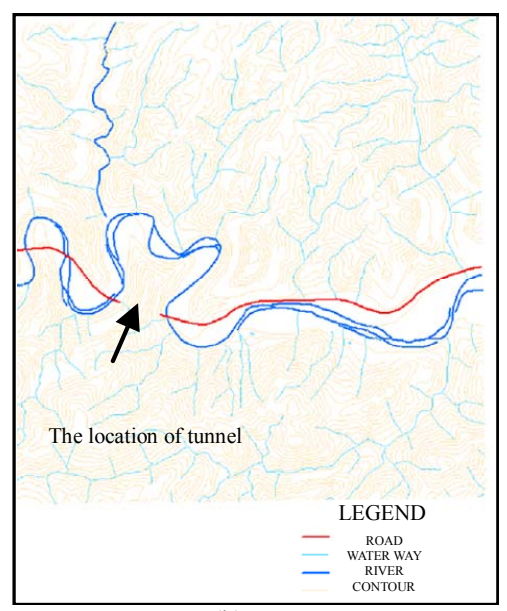

(b)

Figure 1. (a) SPOT image from Tabriz-Miyaneh road in Iran; (b) 1:25,000 topographic map of this region. 


\subsubsection{Image Classification Using Neural Network Method}

Artificial Neural Networks (ANN) is a non-linear model that acts similar to a human neural system. Each ANN consists of a series of nodes and weighted connections between them [12]. A schematic diagram of the structure of an ANN is shown in Figure 2.

In fact nodes are connected by weighted connectors to different layers in the ANN. In ANN there are different layers that can be generally divided into three groups of input layers, hiding layers and output layers [12]. ANN consists of two stages in classification, training and recalling [7]. In the training stage, an operation is being conducted to improve the connection weighting coefficients through some iteration processes. In this training stage the known inputs and outputs are used, and conesquently is called Supervised Classification. Back-Propagation method is based on the concept suggested by Parker and Rumelhart (1980). This method is a gradient iteration algorithm that is being used to minimize the error as is shown in Equation (1) [7].

$$
E=\frac{1}{2} \sum_{j=1}^{L}\left(d_{j}-o_{j}^{M}\right)^{2}
$$

where, $d_{j}$ and $o_{j}$ are desired output and ANN output respectively. Based on the results of Equation (1), the amount of correction for each weight can be calculated [5].

$$
\left\{\begin{array}{l}
\Delta \mathrm{w}_{i, j}=-\eta \frac{\partial E}{\partial \mathrm{w}_{i, j}} \\
\Delta \mathrm{w}_{i, j}(t+1)=\Delta \mathrm{w}_{i, j}+\alpha \Delta \mathrm{w}_{i, j}(t)
\end{array}\right.
$$

Here $\mathrm{W}$ is the weight between two nodes and $\eta$ is a positive constant parameter that is being used for ajustment and is called training rate. $\alpha$ is a momentum factor (usually called stability factor) and can take value

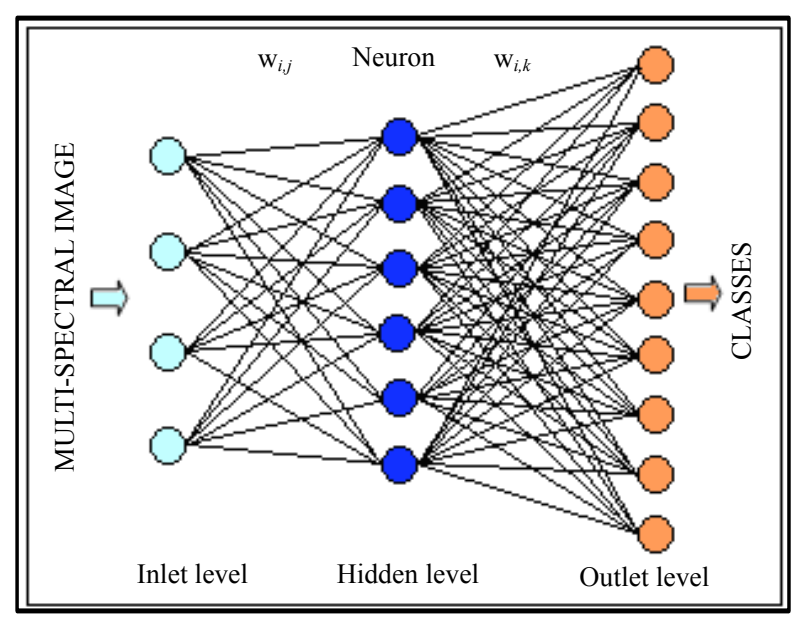

Figure 2. Schematic diagram of the structure of an ANN. between 0 and 1 and $t$ is the number of iterations [12]. In the recalling stage, ANN works according to the trained weighting coefficients, and conducts interpolations and extrapolations. One of the privileges of ANN method compare to traditional statistical methods is that the networks are free in distribution i.e. the training and recalling are depending on the linear combination between data patterns and are independent of input data $[12,13]$. However the reasons for the success of ANN in classification can be summarized as: 1) there is no need for pre-assumption in data distribution; 2) it permits user to make use of the initial knowledge regarding classes and possible limitation; 3) the method is able to manage the spatial data from multi sources and can achieve their classification results equally [13].

\subsubsection{Texture Characteristics}

Neural network inputs have important role for increasing classification accuracy. The spatial resolution of SPOT image is 10 meters. Roads are shown as thin lines. There is River near the road. Rivers in SPOT image has spectral and geometry characteristics like roads. This makes road extraction difficult. For this reason, only by using spectral characteristics, roads cannot be extracted with high precision. Texture information can be used to increase classification accuracy. In this study, the mean and variance and entropy is used as three textures parameters. Variance texture for diagnosis of linear variation plays an important role because in roads, pixels brightness change sharply compared to surrounding pixels. Therefore it can be used to extract road lines from image. There is a relation between Entropy texture and image histogram. In low resolution images, the numbers of road pixels are low. There is also a relation between Entropy texture and image histogram. In low resolution image, the numbers of road pixels are low. Then these pixels have special values in Entropy texture. For this reason Entropy can be used for increasing classification precision. Equation (3) shows how to use the texture entropy on image [14].

$$
E=-\sum_{i=1}^{9} \mathrm{p} \times \log _{2} \mathrm{p}
$$

$\mathrm{P}$ is the Numerical value for each pixel of the image histogram

According to different studies, textures characteristics differ from band to band [7]. In this study, the textures data were produced from SPOT images for each of the bands using ENVI 4.7 software.

\subsection{Classification Performance Using an Artificial Neural Network}

Input data for neural networks contain pixel information in 4 bands and textural characteristics, mean, variance 
and entropy values for each band. The number of input data is 16. As mentioned in Part 3 - 1, training data is required to train an artificial neural network. Training samples play an important role in enhancing the classification accuracy. As is mentioned before, the image has spatial reference. Using topographic map, 47 road pixels and 1342 non-road pixels are used as training data. For designing neural network, different number of layers and neurons are tested in this project. According to Table 1, the best results were seen in the neural network with 4 layers. This network has an input layer with 16 neurons and two hidden layers with 20 neurons in each layer as well as an output layer with single neuron. Learning rate is 0.4 and momentum value is 0.5 . The minimum error is found to be of the order of 0.001 . The number of iteration was 1000 and this network used logisticfunction as activation function.

After training the neural network, the classification algorithm was performed on the entire image where the resulting classified image is shown in Figure 3.

\subsection{Connecting Road Pixels}

To extract the road, the noise created by the image must be eliminated. Mathematical morphology is used to connect road pixels and remove noises. Dilation morphology is used to delete existing gaps in pixels connected and then with using thin morphology, narrow action will be implemented. There are scattered pixels in the image. A filter with a size of 50 in 50 moves over the image. Number of black pixels in each square 50 on 50 is counted. If this number is greater than 40 , the square remains unchanged. But if this number was less than 40 , all pixels in the square would be zero. Type of window that moves over the image and the number of black pixels in each window can be changed according to image spatial resolution. The result of the process is shown in Figure 4.

After this step, those pixels known as road should be connected to each other. An algorithm is proposed here to connect these pixels. A mask with a size of 3 by 3 moves over the image. If the center pixel and one of its neighborhoods has value of 1 , then a vector with four components is formed.

The first two components are the coordinates of the central pixel and two other components are neighboring pixel coordinates. After this step, duplicate vectors are

Table 1. Results of the investigation regarding the number of layers and nodes in ANN.

\begin{tabular}{|c|c|c|c|c|c|}
\hline $\begin{array}{c}\text { Number } \\
\text { of hidden layer }\end{array}$ & $\begin{array}{l}\text { Number of nodes } \\
\text { in hidden layer1 }\end{array}$ & $\begin{array}{l}\text { Number of nodes } \\
\text { in hidden layer } 2\end{array}$ & RMSE & Execution Time & $\begin{array}{c}\text { Number } \\
\text { of iterations }\end{array}$ \\
\hline 1 & 5 & 0 & 0.041 & $245 \mathrm{~s}$ & 1000 \\
\hline 2 & 10 & 0 & 0.034 & $193 \mathrm{~s}$ & 1000 \\
\hline 2 & 5 & 5 & 0.024 & $143 \mathrm{~s}$ & 1000 \\
\hline 2 & 20 & 20 & 0.01 & $86 \mathrm{~s}$ & 1000 \\
\hline
\end{tabular}

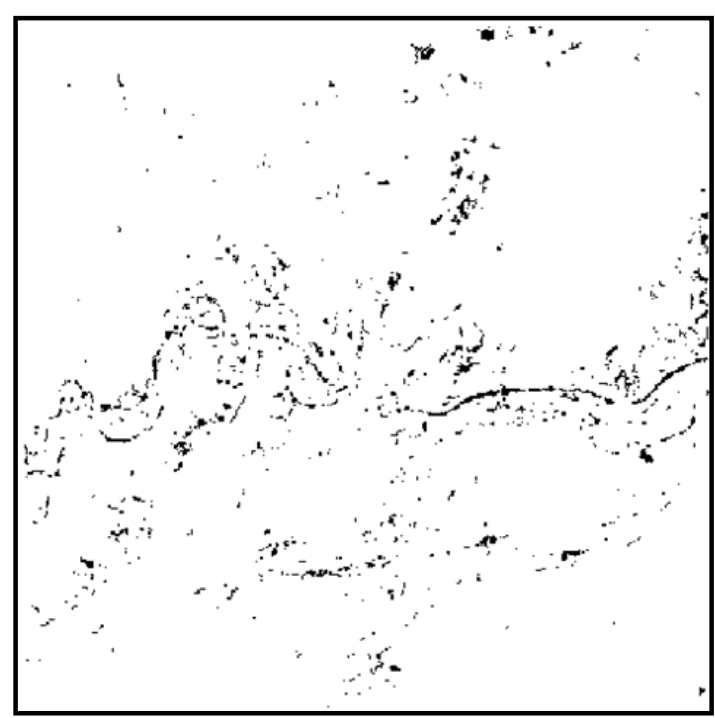

Figure 3. Classified image produced by ANN method.

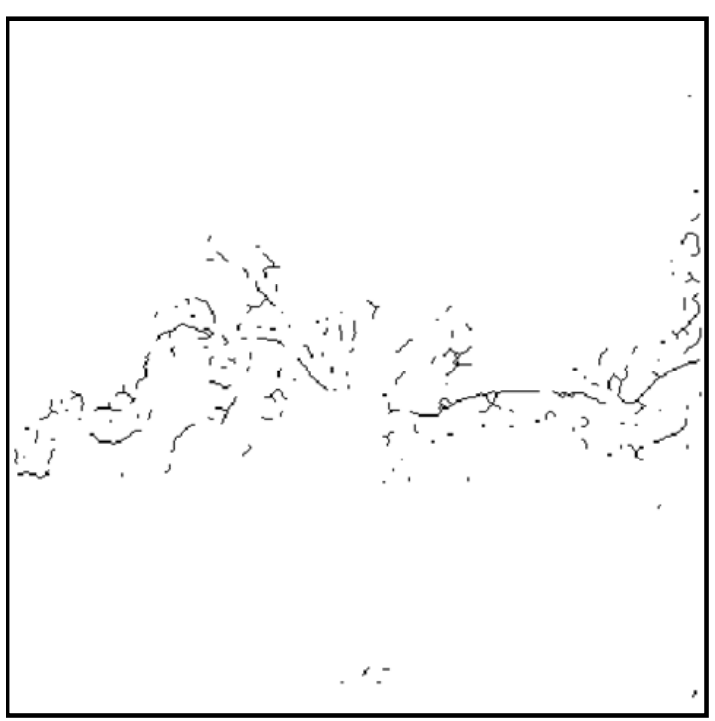

Figure 4. Image produced by remove scattered pixels. 
removed. North and east directions are defined as main direction. With this definition, pixel 1 connects to pixel 2, if the pixel 2 is placed in north or east of the pixel1. Then those vectors which are close to each other with specified threshold (here within 7 pixels) will be connected to each other. Then the next new vectors are connected to each other if the distance between them were less than 10 pixels. In the next move those left alone lines which cannot be classified as road, are removed.

Those lines with the length of less than 50 meters are also removed. After this step, there might still remain some noise. However for removing the rest of noises from the image, digital elevation model can be used. For this the waterways and slope maps can be extracted from digital elevation model. These images are shown in Figure 5.

The fact is that the road pixels cannot be placed in water way and slope value of road is not usually greater than 40 percent. The pixels which are not obeying these conditions are removed. Resulting image is shown in Figure 6.

Those lines that are known as roads should be connected to each other. However it should be noted that if there exists a tunnel, then the road lines will be left disconnected. Here the junctions of roads and mountains have high potential for determination of the location of the tunnels. In this study, junctions of roads and mountains are detected first. For this reason, to connect lines, first start and end of lines are determined. Then a condition is defined where if pixel 1 was end of a line and pixel 2 was the start a line, while the pixel 2 is in the north or east of the pixel 1. Pixels that are existed in line of connection between pixel 1 and pixel 2 are determined. From Each pixel whit 8 neighborhoods, if 5 pixels have slope value greater than 40 percent, then that pixel is shown in red color otherwise that pixel is shown as black color. Then the red pixels that are existed in line of connection between pixel 1 and pixel 2 are counted. If the count of them is greater than 5 , then that pixels are known as tunnel, otherwise the pixels are existed in line of connection between pixel 1 and pixel 2 are known as road. Image is produced by this algorithm shown in Figure 7.

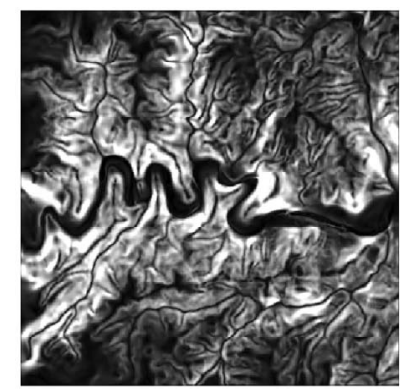

(a)

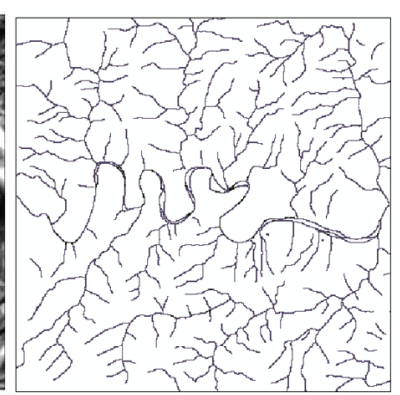

(b)
Figure 5. (a) Waterway image from the study area; (b) Slope image from the study area.

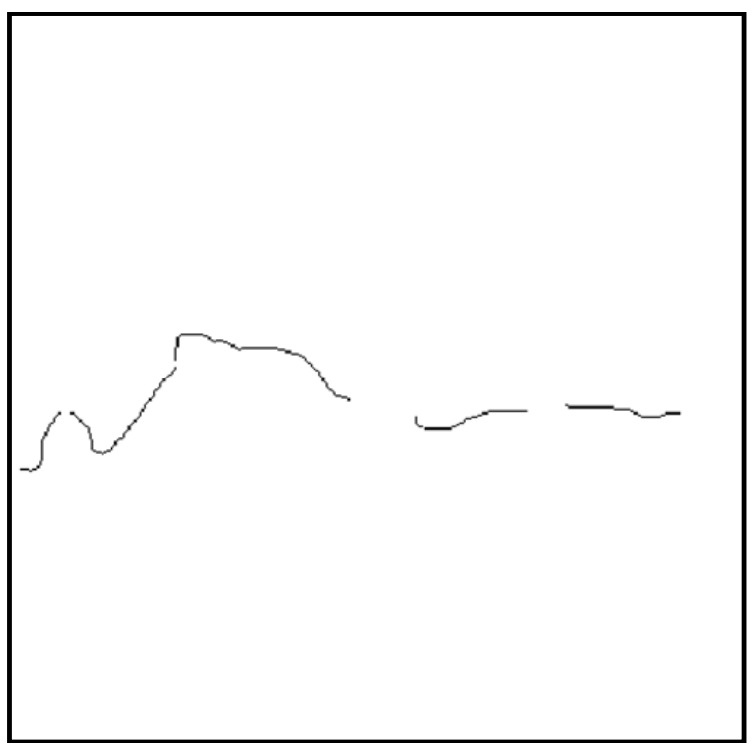

Figure 6. Image produced by connection algorithm and remove road pixel be placed in waterway or that have slope value greater than 40 percent.

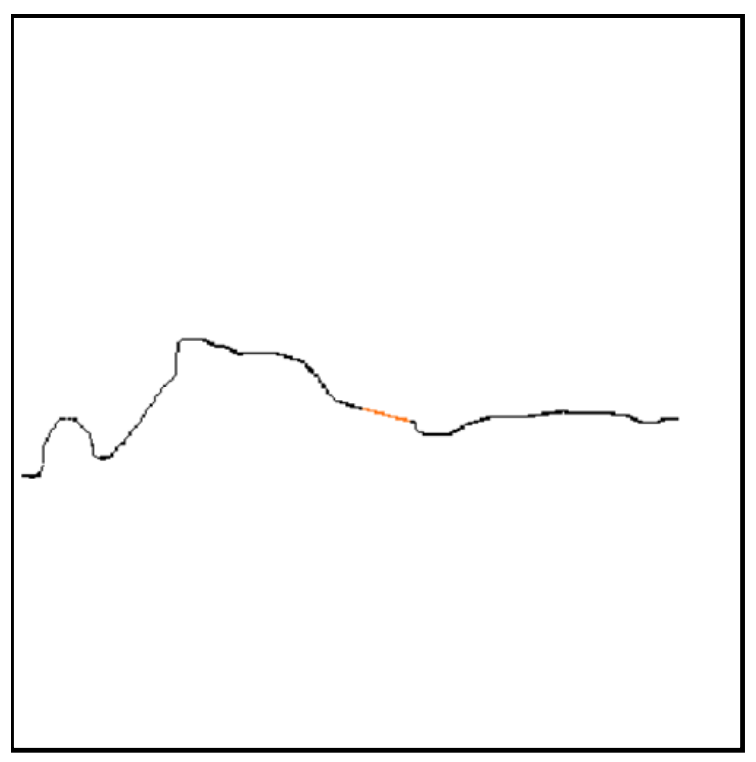

Figure 7. Location of tunnel that is produced by algorithm.

\section{Evaluation of Results}

The road extraction algorithms are evaluated using the confusion matrix (Table 2). To evaluate the results 1244 pixels of roads and 140,508 pixels of non roads as test data were introduced to the algorithm. It should be noted that these data have not been used as training data before.

As can be seen, noise removal, has increased classification precision and real pixels of road are correctly diagnosed. For evaluation of Tunnel extraction, the position of tunnel that extracted by algorithm is shown in Google Map. Figure 8 show that the algorithm has correctly detected the location of tunnel. 
Table 2. Confusion Matrix for road extraction by proposed algorithm.

\begin{tabular}{lccc}
\hline $\begin{array}{l}\text { Overall } \\
\text { accuracy }\end{array}$ & $99 \%$ & & \\
\hline $\begin{array}{l}\text { Kappa } \\
\text { coefficient }\end{array}$ & 0.56 & & \\
\hline Class & Road (test) & Non Road (test) & Total \\
Road & 756 & 488 & 1244 \\
Non road & 636 & 139,872 & 140,508 \\
Total & 1392 & 140,360 & 141,732 \\
Class & Commission (\%) & Omission (\%) & User. Acc. (\%) \\
Road & 46 & 40 & 60 \\
Non Road & 1 & 1 & 99 \\
\hline
\end{tabular}

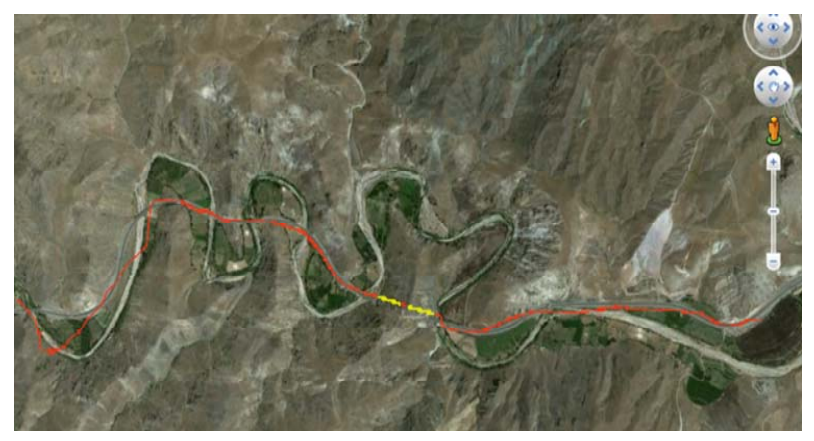

Figure 8. Tunnel extracted from the algorithm is shown in Google earth image.

\section{Conclusion}

Remote sensing is a tool that can be used to indirectly gain valuable information. It should be noted that roads are shown in satellite image in various forms. Algorithms for road extraction are depending upon image spatial resolution. Image classification is one of the methods used for the road extraction. Also use of neural networks is new in image classification. It can increase classification accuracy and decrease the time of processing. In this research, neural networks methods were used for image classification. Input data networks have an important role to increase the classification accuracy. Texture parameters are used to improve classification accuracy. Mathematical morphology is used to remove noises and connection of road pixel. Tunnel location is detected using digital elevation information. Junctions of roads and mountains have high potential for the locating the tunnels. For this reason, in this study, Junctions of roads and mountains are detected.

\section{REFERENCES}

[1] M. Mokhtarzade, M. J. V. Zoej and H. Ebadi, "Automatic
Road Extraction from High Resolution Satellite Image Using Neural Networks, Texture Analysis, Fuzzy clustering and Genetic Algorithms," The International Archives of the Photogrammetry, Remote Sensing and Spatial Information Sciences, Beijing, 2008.

[2] K. D. Bulter, "Detection and Characteristics of Subsurface Cavities, Tunnel and Abandoned Mines," Alion Science and Technology Corporation, Vicksburg, 1996.

[3] J. Mena, "State of the Art on Automatic Road Extraction for GIS Update: A Novel Classification," Pattern Reconition Letters, Vol. 24, No. 16, 2003, pp. 3037-3058. doi:0.1016/S0167-8655(03)00164-8

[4] O. Wiliams, "Geotechnical Explorations for Tunnels and Shafts," 1997.

http://140.194.76.129/publications/eng-manuals/index.ht $\mathrm{ml}$

[5] W. Harvey "Planning and Site Investigation in Tunneling," Premier Business Centers, Belleuve, 1996.

[6] A. Mohammadzadeh, A. Tavakoli and M. J. V. Zoej, "Road Extraction Based on Fuzzy Logic and Mathematical Morphology from Pan-Sharpened IKONOS Images," The Photogrammetric Record, Vol. 21, No. 113, 2006, pp. 4460. doi:0.1111/j.1477-9730.2006.00353.x

[7] M. Mokhtarzade, H. Ebadi and M. J. V. Zoej, "Optimization of Road Detection from High-Resolution Satellite Images Using Texture Parameters in Neural Network Classifiers," Canadian Journal of Remote Sensing, Vol. 33, No. 6, 2007, pp. 481-491. doi:0.5589/m07-055

[8] S. Idbraim, D. Mammass, D. Aboutajdine and D. Ducrot, "An Automatic System for Urban Road Extraction from Satellite and Aerial Images," WSEAS Transactions on Signal Processing, Vol. 4, No. 10, 2008, pp. 563-572.

[9] R. Mangala, "An Effective ANN-Based Classification System for Rural Road Extraction in Atellite Imagery," European Journal of Scientific Research, Vol. 47, No. 4, 2010, pp. 574-585.

[10] J. Mena and J. A. Malpica, "An Automatic Method for Road Extraction in Rural and Semi-Urban Areas Starting from High Resolution Satellite Imagery," Pattern Recognition Letters, Vol. 26, No. 9, 2005, pp. 1201-1220. doi:0.1016/i.patrec.2004.11.005

[11] D. S. Xue and D. Yin, "A Study of Tunnel Entries Automatic Recognition from Super Resolution Remote Sensing Images," University of Science \& Technology of China, Hefei, 2005.

[12] G. Y. Yang, "Geological Mapping from Multi-Source Data Using Neural Networks," M.Sc. Thesis, University of Calgary, Calgary, 1995.

[13] F. Carvajal, "Green House Detection Using Neural Network with a Very High Resolution Satellite Image," PRS Technical Commission VI Symposium, Tokyo, 2006.

[14] R. C. Gonzalez and R. E. Woods, "Digital Image Processing," Addison-Wesley, Boston, 1992. 